\title{
Salud mental en atención primaria en Chile: aprendizajes para Latinoamérica
}

\author{
Mental health in primary care in Chile: lessons for Latin America
}

\author{
Alberto Minoletti ${ }^{1}$, Graciela Rojas², Marcela Horvitz-Lennon³
}

\begin{abstract}
Resumen
El presente artículo describió la experiencia chilena de los últimos 20 años con la integración de salud mental en atención primaria de salud y analizó los resultados de algunas evaluaciones, con el fin de contribuir a los procesos de aprendizaje de cómo disminuir brechas de atención. El retorno a la democracia después de la Dictadura Militar y la Declaración de Caracas fueron factores contribuyentes, así como el alto desarrollo de la atención primaria de salud en Chile y la formulación específica de planes nacionales de salud mental, que incluían la atención primaria de salud como un elemento central. La voluntad política de integración debe expresarse también en la asignación de fondos adicionales para salud mental en atención primaria de salud. Otro factor crítico para la salud mental en atención primaria de salud es la generación de mecanismos de articulación y apoyo entre profesionales de atenciones secundaria y primaria. Se destaca que los indicadores disponibles señalan que Chile habría logrado que la salud mental se transforme en un componente integral de su atención primaria de salud, posiblemente de forma sustentable, aunque aún existen muchas interrogantes que requieren estudios específicos de mayor profundidad.
\end{abstract}

Palabras clave: servicios de salud mental; atención primaria de salud; salud mental; psiquiatría.

\begin{abstract}
This article described the integration of mental health into primary care services in Chile over the past 20 years and analyzed the results of a few evaluations. The purpose was to contribute to the processes of learning how to reduce mental health care gaps throughout Latin America. The return to democracy after the Military Dictatorship and the Caracas Declaration were contributing factors to the integration, as were the high development of the Chilean primary care system and the development of national mental health plans that included primary care services as a central element. The political will of integration should also include the assignment of additional funds for mental health, within the primary care system. Another critical factor for mental health in primary care is the generation of mechanisms for coordination and support between secondary and primary care professionals. The article emphasized that available indicators show that Chile has successfully made mental health a central component of primary care services, possibly for the long term, but there are many remaining questions that require further studies.
\end{abstract}

Keywords: mental health services; primary health care; mental health; psychiatry.

Trabajo realizado en la Escuela de Salud Pública, Facultad de Medicina, Universidad de Chile - Santiago de Chile, Chile.

1Profesor en la Escuela de Salud Pública, Facultad de Medicina, Universidad de Chile; Ex-jefe del Departamento de Salud Mental, Ministerio de Salud,

Gobierno de Chile - Santiago, Chile.

2Profesora en el Hospital Clínico, Facultad de Medicina, Universidad de Chile - Santiago, Chile.

${ }^{3}$ Physician Scientist, RAND Corporation - Pittsburgh PA, USA.

Dirección para correspondencia: Alberto Minoletti - Escuela de Salud Pública, Facultad de Medicina, Universidad de Chile - Avenida Independencia, 939 -

CEP: 8380453 - Santiago, Chile - E-mail: aminoletti@med.uchile.cl

Soporto financiero: ningun.

Conflicto de interese: nada a declarar. 


\section{INTRODUCCIÓN}

El Plan de Acción sobre Salud Mental aprobado por los ministros de la salud de las Américas, en el Consejo Directivo de la Organización Panamericana de la Salud de la Organización Mundial de Salud (OPS/OMS) del año 2009, reconoce los servicios de salud mental centrados en la atención primaria de salud (APS) como una de las cinco estrategias fundamentales para contribuir a que la población de las Américas disfrute de una óptima salud mental ${ }^{1}$. Los fundamentos de esta estrategia se relacionan tanto con la necesidad de disminuir la brecha de tratamiento de trastornos mentales, a través de la participación de equipos de salud general, como en la adopción de un enfoque integral de salud que facilite las interrelaciones entre aspectos físicos y mentales. Este enfoque es consonante con las orientaciones técnicas que ha elaborado la OMS en los últimos años ${ }^{2,3}$.

La incorporación de la salud mental a la APS no es un concepto nuevo. Ya en la Declaración de Alma Ata, adoptada en la Conferencia de 1978, patrocinada por la OMS y UNICEF, se llamaba a la transformación de los sistemas de atención tradicionales, señalando que la APS era la forma más efectiva de alcanzar un nivel adecuado de salud entendido como el estado de completo bienestar físico, mental, y social ${ }^{4}$. La importancia de esta declaración se encuentra en el reconocimiento histórico que la APS tenía el potencial de mejorar los problemas de salud del mundo, ya sean de tipo físico o mental.

La Conferencia Regional para la Restructuración de la Atención Psiquiátrica en Latinoamérica, organizada por OPS/OMS en Caracas en 1990, tuvo una influencia mayor en las políticas de salud mental de la región. En ella se formularon los principios que han servido de modelo conceptual para la Reforma Psiquiátrica, la cual fue desarrollada en los años siguientes en Latinoamérica ${ }^{5,6}$. En esa Declaración, participantes de diversos países expresaron su compromiso de transformar la atención basada en hospitales psiquiátricos por sistemas basados en la comunidad, considerando que la APS representaba el principal vehículo para la entrega de servicios de salud mental. La alta motivación generada en Caracas fue canalizada por OPS/OMS a través de la Iniciativa para la Restructuración de los Servicios Psiquiátricos, cuyo objetivo fue la promoción de iniciativas de salud mental comunitaria. Este proceso se vió favorecido por el coincidente término de dictaduras militares en varios países de la región en las dos décadas precedentes. Algunos países aprobaron leyes y muchos formularon políticas basadas en los principios de Caracas, incluyendo como componentes fundamentales, en su mayoría, estrategias de mejora de acceso a través de la inclusión de la salud mental en la APS7.

Entre los países latinoamericanos que han sido destacados por la integración de salud mental en APS, nacional o regional, se mencionan a Argentina, Brasil, Cuba, Chile, El Salvador, Nicaragua, Guatemala, y Panamá2, ${ }^{27}$ Es de interés evaluar y analizar éstas buenas prácticas con el fin de generar procesos de aprendizaje que contribuyan a implementar el Plan de Acción sobre Salud Mental de la región y así responder mejor a las enormes necesidades de nuestras poblaciones. En este sentido, el presente artículo describió la experiencia chilena de los últimos 20 años con la integración de salud mental en APS y analizó los resultados de algunas evaluaciones.

\section{PROCESO DE INTEGRACIÓN DE LA SALUD MENTAL EN ATENCIÓN PRIMARIA DE SALUD EN CHILE}

La primera vez que se conceptualizó formalmente la idea de los servicios de salud mental comunitarios y de integración en APS fue en 1966, con el primer programa nacional de salud mental ${ }^{8}$. Aunque este programa nunca llegó a ser implementado por falta de apoyo político y de recursos, representó un hito histórico significativo al concertar a un grupo de profesionales planteando estrategias de salud pública para enfrentar la alta prevalencia de enfermedades mentales, observada en estudios epidemiológicos. En este programa se señalaba que los hospitales psiquiátricos eran inefectivos y de alto costo para responder a las necesidades de salud mental, proponiéndose en cambio el desarrollo de una red de servicios basada en APS y en hospitales generales.

Uno de los autores de este primer programa, el profesor Juan Marconi, lideró un grupo universitario que se trasladó desde el principal hospital psiquiátrico del país al sector Sur de Santiago, en 1968, con el objeto de desarrollar una experiencia piloto de psiquiatría comunitaria. Junto con brindar atención clínica en un centro de APS y en un hospital general, se capacitaron equipos de APS en el tratamiento del alcoholismo y se promovió la activa participación de la comunidad en la solución de sus problemas de salud mental de mayor prevalencia (alcoholismo, neurosis, y retraso del desarrollo cognitivo infantil $)^{9}$. Esta experiencia piloto fue diseminada en pequeña escala a otros lugares de Chile, especialmente en alcoholismo, a través de APS y grupos de autoayuda ${ }^{9,10}$.

Como muchas otras iniciativas comunitarias y de salud pública, las experiencias descritas fueron clausuradas por la Dictadura Militar en 1973, y muchos de sus líderes fueron despedidos del sistema público, exiliados, detenidos y algunos fueron asesinados. Las únicas iniciativas que sobrevivieron fueron los servicios de psiquiatría en hospitales generales, el tratamiento de la dependencia de alcohol en centros de APS y la disponibilidad en ellos de medicamentos psicotrópicos básicos (clorodiazepóxido, clorpromazina, haloperidol, amitriptilina e imipramina) $)^{8,9}$. 
Con el retorno a la democracia en 1990, se produjo en Chile una marcada modificación del énfasis de las políticas de salud. Se tomaron diversas medidas para el reforzamiento de la red pública, aumentando la inversión en infraestructura y los presupuestos operacionales, y mejorando las competencias técnicas de sus equipos y la coordinación de los establecimientos. Desde mediados de la década del 1990, se inició un activo proceso de análisis y propuestas que culminaría al cabo de diez años con el establecimiento de una profunda reforma de salud.

$\mathrm{Al}$ mismo tiempo, el nuevo gobierno mostró una sensibilidad mayor hacia aspectos psicológicos y sociales de la salud. Fruto de esto fue la constitución de un equipo multidisciplinario de ocho profesionales a cargo de la salud mental en el Ministerio de Salud, la conformación de una red nacional de profesionales encargados de salud pública en este tema y los apoyos político y financiero para iniciar gradualmente programas comunitarios innovadores ${ }^{8,11}$.

Por otra parte, la coincidencia de que a los pocos meses de que asumiera el nuevo gobierno democrático se efectuara la Conferencia de Caracas contribuyó a dar mayor impulso a los programas comunitarios y en especial a aquellos basados en APS. En Chile, la Declaración fue ampliamente difundida entre profesionales y técnicos y analizada en varias reuniones masivas ${ }^{12}$.

La primera política y plan nacional de salud mental fueron oficialmente promulgadas en 1993 por el ministro de salud. El plan incluyó la salud mental en APS como una de las seis prioridades ${ }^{13}$. Muchos profesionales de APS (médicos generales, enfermeras, y asistentes sociales) fueron capacitados para atender problemas de salud mental, tales como trastornos emocionales y violencia doméstica, así como a realizar intervenciones preventivas de salud mental en escuelas básicas. Además, se mejoró la coordinación entre APS y otros niveles de atención a través de reuniones periódicas de representantes de los distintos dispositivos de salud mental de cada área sanitaria.

La primera etapa de integración de salud mental en APS se realizó en su mayor parte con los recursos que se disponían para la generalidad de los problemas de salud. Una mínima cantidad de fondos adicionales específicos para salud mental fueron destinados en forma intermitente para la mayoría de los centros de APS, los que fueron utilizados principalmente en capacitaciones y materiales educativos para usuarios y equipos de salud. En 1992, 60 centros de APS fueron reforzados con tres profesionales con media jornada, habitualmente psicólogo, trabajador social y matrona (enfermera obstétrica), con el fin de realizar actividades psicosociales.

Además del desarrollo de servicios de salud mental en APS, el Plan de Salud Mental de 1993 contribuyó al aumento de equipos de especialidad ambulatorios y a la instalación de los primeros hospitales de día, programas de rehabilitación psicosocial basados en la comunidad y hogares protegidos.

Si bien las acciones de salud mental implementadas en APS en este período no lograron una cobertura significativa, sirvieron como proyectos pilotos (aunque en su gestación no fueron pensados como tales), generando procesos de aprendizaje de gran utilidad para el desarrollo posterior. Así por ejemplo, la incorporación de psicólogos al equipo de APS fue considerada como positiva y a partir del año 2000 fue establecida como una política nacional. Varios de los aspectos insuficientes del primer plan, tales como definición ambigua del rol de la APS en salud mental, imprecisión en la cantidad de recursos necesarios, y falta de apoyo de especialistas a los equipos generales, fueron también insumos importantes.

Después de seis años de implementación de este plan, se produjo un consenso creciente de la necesidad de formular uno nuevo que recogiera los nuevos aprendizajes junto con la evidencia que se estaba generando en otros países, que transformara el modelo de psiquiatría institucional por uno comunitario y especificara los recursos necesarios para abordar los problemas de salud mental de mayor prioridad en el país. En los años 1998 y 1999, el equipo de salud mental del Ministerio de Salud en conjunto con organizaciones de familiares y de profesionales realizó una serie de acciones de abogacía a favor del modelo comunitario y de mayores recursos para salud mental. En un proceso de amplia participación de familiares, usuarios y profesionales, un segundo plan nacional fue formulado en 1999 y se inició su implementación al año siguiente ${ }^{14}$, coincidente con el inicio del tercer gobierno democrático de la coalición política de centro-izquierda, que sucedió a la Dictadura.

El segundo plan de salud mental definió un conjunto coherente de estrategias para contribuir a mejorar la salud mental de los chilenos ${ }^{15}$, incluyendo tanto acciones del sector salud como las de otros sectores del Estado, desde lo promocional y preventivo a lo curativo y de rehabilitación, aspectos programáticos de cómo abordar las prioridades y de servicios de salud para el desarrollo de redes de dispositivos territoriales, el rol de las organizaciones de usuarios y familiares y el de los equipos de salud, elementos de regulación y calidad y de financiamiento y recursos. En cada una de estas estrategias se definieron aspectos relevantes de la salud mental en APS (Tabla 1).

De los seis problemas prioritarios definidos en el plan, solo tres - depresión, esquizofrenia y abuso/dependencia de alcohol y drogas - han recibido apoyo político y financiamiento suficientes como para lograr servicios disponibles y accesibles a lo largo de Chile. Estos tres han sido también incluidos en el sistema de garantías explícitas de salud (GES) desde el 2004, como parte de la reforma de salud. De acuerdo 
a la ley de GES, tanto el seguro público (cobertura del $74 \%$ de la población) como los privados (el 16\% de la población) deben ofrecer un conjunto de garantías de acceso, calidad, oportunidad y protección financiera para 56 enfermedades prioritarias $^{16}$. Estos tres problemas de salud mental han sido integrados en grados diversos en APS:

- la mayor integración ha sido para depresión, donde más del $80 \%$ de las personas mayores de 15 años pertenecientes al seguro público son tratadas en APS por médicos generales y psicólogos. Este programa fue iniciado en unos pocos centros de APS en el año $2001 \mathrm{y}$ luego progresivamente a los 520 existentes en el país en el curso de cinco años ${ }^{17}$;

- la integración del manejo del abuso/dependencia de alcohol y drogas es intermedia, con alrededor del 50\% de las personas con estos problemas siendo tratados en atención primaria, principalmente en caso de abuso y en dependencias leves;

- el rol de la atención primaria en esquizofrenia se limita solo a la detección de primeros episodios y referencia a un psiquiatra.

Las acciones de promoción de salud mental y prevención de enfermedades mentales planteadas en el plan nacional han tenido un bajo nivel de implementación, debido a la falta de apoyos político y financiero. Una oportunidad interesante surgió a partir del 2007, con la puesta en marcha de un programa de desarrollo infantil temprano llamado "Chile Crece Contigo"18. Este programa es una iniciativa intersectorial liderada por el Ministerio de Planificación Nacional (actualmente transformado en Ministerio de Desarrollo Social) y en el que participan también los Ministerios de Educación y Salud, y su objetivo es facilitar el desarrollo psíquico y físico desde el embarazo hasta los cuatro años, reduciendo inequidades y promoviendo los derechos de los niños. La puerta de entrada es la APS, y se espera que el aumento de actividades educativas sobre crianza, visitas domiciliarias a familias en riesgo y acceso a salas cunas y jardines infantiles, entre otras prestaciones, contribuya a mejorar la salud mental de la población con mayor vulnerabilidad social.

El proceso de integración de la salud mental en APS desde el 2000 hasta la conclusión de este estudio no ha estado exento de dificultades ${ }^{12}$. Los profesionales de salud mental, tanto en el área de salud pública como en clínica, han tenido que invertir un tiempo considerable en abogacía, tratando de convencer a autoridades de salud desde el nivel nacional hasta las localidades más pequeñas de las bondades de esta integración, especialmente por el predominio biomédico y curativo en el sistema de salud chileno. Por otra parte, el GES, con su fuerte focalización en unos pocos problemas prioritarios, genera inequidades para las personas que no cumplen con los criterios de inclusión a pesar de sufrir enfermedades severas y discapacitantes. A su vez, el énfasis desmedido del sistema público en evaluar programas por el número de personas atendidas y/o prestaciones entregadas atenta contra la calidad de atención.

\section{EVALUACIÓN DEL PROCESO DE INTEGRACIÓN}

La salud mental integrada en APS ha llegado a ser parte oficial de las políticas de salud pública en Chile. Es así como la recientemente formulada Estrategia Nacional de Salud 2011-2020, que ha incorporado como una de las metas la disminución de discapacidad en personas con enfermedad mental y la utilización de la estrategia de la detección temprana y el tratamiento oportuno en APS $^{19}$. En dicho documento se destaca la expansión del Programa Integral de Salud Mental en la Atención Primaria a partir del 2006, el cual incorpora la atención en depresión, violencia intrafamiliar, consumo de alcohol y otras drogas, trastornos hipercinéticos, maltrato

Tabla 1. Plan Nacional de Salud Mental de Chile del año 200014. Principales estrategias relacionadas con la incorporación de salud mental a la atención primaria de salud

\begin{tabular}{ll} 
Estrategias & \multicolumn{1}{c}{$\begin{array}{c}\text { Especificaciones } \\
\text { Priorización de problemas de mayor prevalencia y } \\
\text { discapacidad }\end{array}$} \\
$\begin{array}{l}\text { Definición de actividades para atenciones primaria } \\
\text { y secundaria }\end{array}$ & $\begin{array}{l}\text { Trastorno hipercinético/de la atención, víctimas de violencia doméstica y de la } \\
\text { dictadura, depresión, esquizofrenia, abuso/dependencia de sustancias y demencia. } \\
\text { Programa de salud mental que define el tipo de prestaciones a entregar a cada nivel } \\
\text { de atención y por cada problema prioritario. } \\
\text { Nivel de resolución en atención primaria de salud }\end{array}$ \\
$\begin{array}{l}\text { APS como puerta de entrada única y capaz de resolución de trastornos leves y } \\
\text { moderados (se estimó el porcentaje de casos a resolver por cada nivel de atención) } \\
\text { Articulación de los equipos de atención primaria de salud con los de atención }\end{array}$ \\
$\begin{array}{l}\text { Consultoría de salud mental } \\
\text { secundaria, a través de visitas mensuales de los segundos para revisar, en conjunto, } \\
\text { casos de mayor dificultad. }\end{array}$ \\
$\begin{array}{l}\text { Estimación de la demanda esperada, número de prestaciones necesarias, horas } \\
\text { de miembros del equipo de atención primaria de salud requeridas y cantidad de } \\
\text { medicamentos a usar. }\end{array}$
\end{tabular}


infantil, y trastornos emocionales o conductuales de la infancia y adolescencia. Además, señala que se ha aumentado la cobertura de tratamiento de trastornos mentales, pasando de una población bajo control de aproximadamente 245.000 personas en el 2005 a cerca de 560.000 en el 2010.

La voluntad política de integración se ha expresado también en la asignación de fondos para salud mental en APS, aumentando desde casi cero en 1990 a 0,9\% del presupuesto de salud en el sector público en el 2009 (Figura 1). Este hecho contrasta con la disminución del peso presupuestario de los hospitales psiquiátricos, el cual ha bajado desde 0,86 a $0,59 \%$ entre los años 2002 y 2009 (Figura 1). La mayor parte de los fondos para APS se han destinado a psicólogos, quienes han sido contratados en todos los centros urbanos y en muchos de los equipos de salud móviles que visitan las postas de salud rurales, así como al mejoramiento de medicamentos, incorporando, por ejemplo, fluoxetina, sertralina, y venlafaxina.

Alrededor de un tercio de las consultas de salud mental que se realizan en APS son efectuadas por médicos generales (Figura 2), focalizándose principalmente en los aspectos biológicos y en el tratamiento farmacológico. Los psicólogos se han responsabilizado de la atención psicosocial, concentrando más del 50\% del trabajo en salud mental. Los trabajadores sociales y otros profesionales han dejado gradualmente de hacer consultas de salud mental, y entre los años 2002 y 2010 disminuyeron su participación desde el 26,8 al 11,7\% (Figura 2). La participación de técnicos y usuarios y familiares en APS ha sido minoritaria en comparación con los profesionales, tal como se observó en la evaluación WHO-AIMS del $2006^{20}$.

La mayor disponibilidad de servicios de salud mental en APS se refleja en la mayor capacidad para entregar consultas de salud mental a la población del seguro público. Así, la tasa de consultas por médicos de APS ha aumentado desde ser casi inexistentes dos décadas atrás a más de 60 consultas por 1.000 beneficiarios en los últimos años, con una velocidad de crecimiento muy superior al experimentado por la tasa de consultas psiquiátricas (Figura 3).

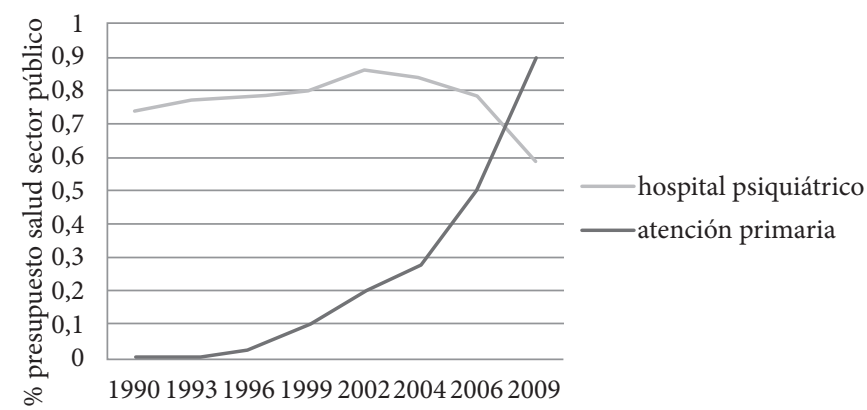

Figura 1. Porcentaje del presupuesto de salud en el sector público asignado a salud mental en atención primaria y hospitales psiquiátricos en Chile: 1990 a 2009 (fuente de los datos: Ministerio de Salud)
La accesibilidad a tratamiento de personas con diversos trastornos mentales también ha aumentado considerablemente en APS, lo cual se ejemplifica con las tasas de ingresos (Figura 4). Sin embargo, a partir del 2008 se apreció una disminución de la misma para algunos trastornos y en particular para los depresivos.

Las únicas evaluaciones de resultados de tratamientos en atención primaria que se han efectuado en Chile son las relativas a depresión. Un primer estudio demostró que un modelo de cuidados progresivos, en mujeres con depresión mayor, ejecutado por trabajadores sociales, enfermeras y médicos generales fue más efectivo que el tratamiento habitual y solo levemente de mayor costo ${ }^{21,22}$, entregando insumos importantes para el desarrollo del programa nacional de depresión.

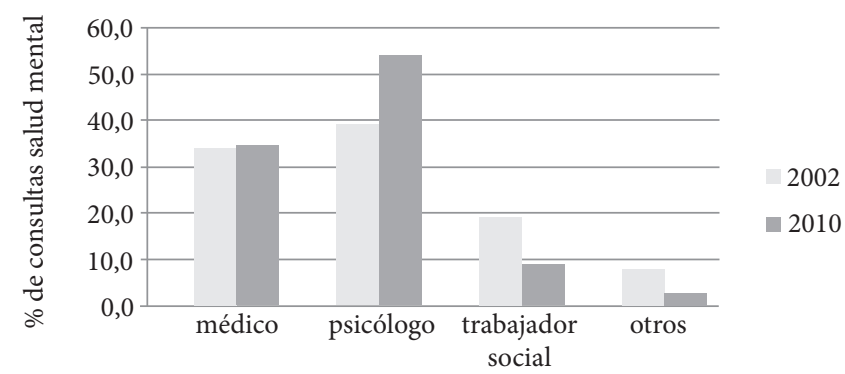

Figura 2. Porcentaje de consultas de salud mental realizadas por distintos profesionales en atención primaria en Chile en los años 2002 y 2010 (fuente de los datos: Ministerio de Salud)

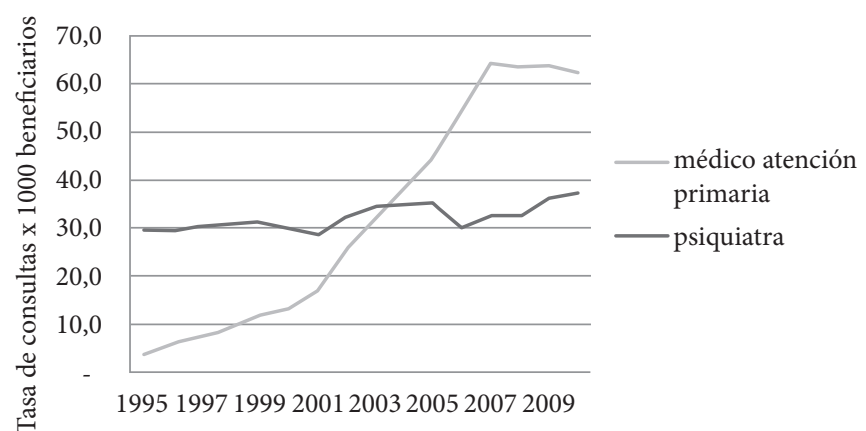

Figura 3. Tasa de consultas de salud mental anuales a médicos de atención primaria y a psiquiatras en Chile 1995 a 2010 (fuente de los datos: Ministerio de Salud)

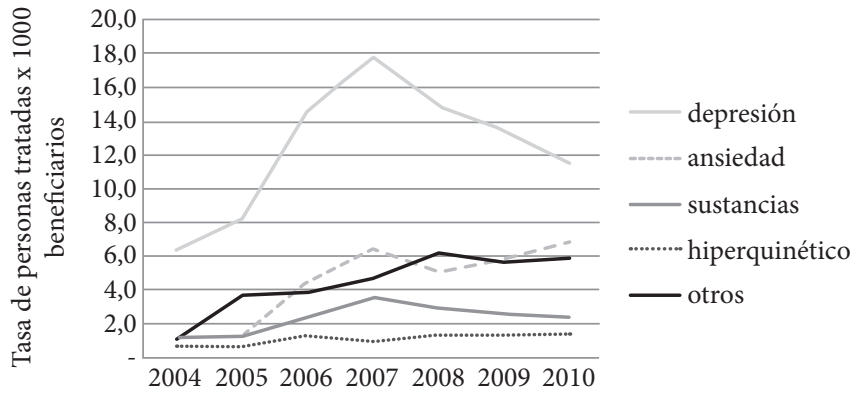

Figura 4. Tasa de personas que ingresan anualmente a tratamiento en atención primaria por distintos trastornos mentales en Chile 2004 a 2010 (fuente de los datos: Ministerio de Salud) 
Una evaluación realizada, cuando el programa se encontraba en un nivel medio de desarrollo, concluyó que el tratamiento era efectivo para disminuir síntomas depresivos y de comorbilidad, con efectos favorables tanto en las consultantes como en sus parejas, pero que un nivel no despreciable de abandono de tratamiento atentaba contra su efectividad ${ }^{23,24}$. Finalmente, otro estudio mostró que la detección y el tratamiento multicomponente de la depresión postparto en atención primaria no solo es posible sino que efectiva, mostrando resultados significativamente superiores a los cuidados habituales ${ }^{25}$; información que fue utilizada para mejorar la calidad del programa nacional.

\section{DISCUSIÓN}

Los antecedentes presentados indican que Chile ha logrado que la salud mental se transforme en un componente integral de su APS, posiblemente de forma sustentable en el tiempo y que ello estaría produciendo algunos beneficios a su población. Futuros estudios deberán dimensionar si esta integración se ha producido de forma e intensidad adecuadas para el nivel de recursos de este país y de las necesidades de su población. También será necesario determinar si la calidad de los servicios cumple con los estándares nacionales e internacionales y si ellos producen los resultados esperados en las personas.

Los avances descritos en la integración de salud mental a APS no ocurrieron como un fenómeno aislado, sino que se dieron en un contexto de desarrollo general del sistema público de salud y específico de la red de salud mental ${ }^{8,11,15,20,26,27}$.

La contribución que pudieran tener los aprendizajes ocurridos en Chile para otros países latinoamericanos fue analizada de acuerdo a los siguientes interrogantes.

¿Se requeriría un cierto nivel de desarrollo de Atención

Primaria de Salud en salud física antes de integrar la salud mental?

La integración chilena ocurrió en una APS con un alto grado de desarrollo, aunque esto no parece ser un requisito indispensable. Si bien esto condicionó algunos aspectos favorecedores, como el disponer de infraestructura y equipos en todo el territorio nacional y de un modelo de salud pública exitoso, al mismo tiempo contribuyó a generar algunas barreras, como es el caso del modelo biomédico que no es fácil de modificar después de 50 años de implementación. Se podría pensar que, de acuerdo al desarrollo conceptual y de prácticas que disponemos en la actualidad, cualquier grado de desarrollo de APS permitiría algún nivel de integración de salud mental.

\section{¿Qué tan necesario es tener una política y/o un plan}

nacional para esta integración?

Probablemente sería muy difícil alcanzar resultados en gran escala si no se dispone de algún documento oficial que explicite la prioridad de la integración de la salud mental, especialmente si se considera la larga lista de problemas sanitarios y sociales que compiten entre sí para alcanzar un lugar en la agenda política. La experiencia chilena sugiere también que el plan nacional necesita una clara definición del rol que va a cumplir la APS en salud mental y de la forma de articulación con el resto de la red de servicios de salud mental. Además del plan, el disponer de guías clínicas para el manejo de problemas de salud mental en APS puede contribuir a disminuir algunas resistencias de los equipos locales.

\section{¿Es imprescindible disponer de recursos adicionales} para la integración de la salud mental?

De acuerdo a lo descrito, los recursos adicionales parecen imprescindibles para superar el recargo laboral y la insuficiencia de recursos que tiene la APS en países de ingresos bajos y medios. La construcción de paquetes de tratamiento, basados en guías clínicas y en los que se especifican tipo y cantidad de prestaciones y los recursos que ellas involucran, contribuyeron en el caso de Chile a dimensionar la cantidad de recursos necesarios y a facilitar la asignación de recursos.

\section{¿Se puede integrar la salud mental a Atención Primaria} de Salud si no se disponen de psicólogos?

Existen varios países que han desarrollado formas exitosas de integración sin la participación de psicólogos ${ }^{2}$. La decisión política chilena de utilizar psicólogos no obedeció tanto a un análisis conceptual o basado en evidencias, sino más bien a un fenómeno coyuntural de alta oferta de estos profesionales y de abogacía corporativa. El riesgo de incorporar especialistas en APS es que el equipo de salud general disminuye su involucramiento en salud mental (Figura 2). Una de las debilidades del proceso chileno, y que ha encarecido su aplicación, es haber utilizado casi exclusivamente profesionales en APS con una baja utilización del personal técnico y muy insuficiente participación de usuarios y familiares.

¿Cuál es el rol de los equipos secundarios de salud mental en Atención Primaria de Salud?

Una de las actividades críticas para el proceso de integración ha sido la visita periódica de profesionales secundarios, especialmente psiquiatras, a los centros de APS. Esta actividad, que en Chile se ha denominado "Consultoría de Salud Mental", ha permitido que los médicos de APS desarrollen la capacidad técnica y la confianza para tratar personas con trastornos mentales, 
además de establecer una alianza de trabajo con el equipo especializado, articulando de manera más fluida y permitiendo negociar las naturales diferencias entre los dos niveles.

\section{CONCLUSIONES}

La experiencia de integración de salud mental a APS en Chile demuestra que este proceso es posible en países latinoamericanos, y que en un escenario de insuficientes recursos puede contribuir en forma importante a mejorar disponibilidad y accesibilidad. Entre los aprendizajes que ha generado esta experiencia para la región, se destacan el papel facilitador que puede jugar una política $y / o$ plan nacional de salud mental, la necesidad de destinar recursos adicionales que sustenten las prestaciones de salud mental en APS y la generación de mecanismos de articulación y apoyo entre profesionales de atenciones secundaria y primaria.

\section{REFERENCIAS}

1. Organización Panamericana de la Salud. Resolución CD 49.R17. Estrategia y plan de acción sobre salud mental. Washington, DC: OPS; 2009.

2. Funk M, Ivbijaro G. Integrating Mental Health into Primary Care: a Global Perspective. Geneva: World Health Organization and World Organization of Family Doctors; 2008.

3. Organización Mundial de la Salud. Guía de Intervención mhGAP para los trastornos mentales, neurológicos y por uso de sustancias en el nivel de atención de la salud no especializada. Versión 1.0. [cited 2012 Oct 25]. Ginebra: OMS; 2011. Available from: http://whqlibdoc.who.int/ publications/2011/978.924.3548067_spa.pdf

4. World Health Organization and United Nations Children's Fund. Declaration of Alma-Ata. International Conference on Primary Health Care. [cited 2012 Oct 25]. Alma-Ata, USSR; 1978. Available from: http:// www.paho.org/spanish/dd/pin/alma-ata_declaracion.htm

5. González R, Levav I. Reestructuración de la Atención Psiquiátrica. Bases Conceptuales y Guías para su Implementación. HPA/MND I.9I. Washington, DC: Organización Panamericana de la Salud; 1991.

6. Levav I, Restrepo H, Guerra de Macedo C. The restructuring of psychiatric care in Latin America: A new policy for mental health services. J Public Health Pol. 1994;15(1):71-85.

7. Caldas de Almeida JM, Horvitz-Lennon M. Mental health care reforms in Latin America: An overview of mental health care reforms in Latin America and the Caribbean. Psychiatr Serv. 2010;61(3):218-21.

8. Minoletti A, Rojas G, Sepúlveda R. Notas sobre la Historia de las Políticas y Reformas de Salud Mental en Chile. En: Armijo MA (Ed). La Psiquiatría en Chile. Apuntes para una Historia. Santiago, Chile: Laboratorios Royal Pharma; 2010; p. 132-55.

9. Marconi J. Política de salud mental en América Latina. Acta Psiquiatr Psicol Am Lat. 1976;22:112-8.

10. Minoletti A, Pemjean A. Salud mental en el norte de Chile: Un desafío Teórico y Operacional. Acta Psiquiatr Psicol Am Lat. 1973;19:434-44.

11. Farmer CM. Chile: Reforms in national mental health policy. In: Caldas de Almeida JM, Cohen A (Eds). Innovative Mental Health Programs in Latin America \& the Caribbean. Washington, DC: Pan American Health Organization (PAHO/WHO); 2008. p. 44-61.
12. Minoletti A, Rojas G, Horvitz-Lennon M. Integrating Mental Health into Primary Care: Lessons from the Experience of Chile. In: Sorel E (Ed.). $21^{\text {st }}$ Century Global Mental Health. Jones \& Bartlett Learning; 2012; p. 255-81.

13. Chile. Ministerio de Salud, División de Programas de Salud. Políticas y Plan Nacional de Salud Mental. Santiago: Ministerio de Salud; 1993.

14. Chile. Ministerio de Salud, Unidad de Salud Mental. Plan Nacional de Salud Mental y Psiquiatría. Santiago: Ministerio de Salud; 2000.

15. Minoletti A, Zaccaria A. Plan nacional de salud mental en Chile: 10 años de experiencia. Rev Panam Salud Pública. 2005;18(4/5):346-58.

16. World Bank. Realizing Rights through Social Guarantees: an analysis of new approaches to social policy in Latin America and South Africa (Updated February 01, 2008). [cited 201210 25]. Available from: http:// www-wds.worldbank.org/external/default/WDSContentServer/WDSP/ IB/2008/03/27/000333037_200.803.27040951/Rendered/PDF/400470W P0P10371gh0Social0Guarantees.pdf

17. Araya R, Alvarado R, Minoletti A. Chile: an ongoing mental health revolution. Lancet. 2009;374:597-8.

18. Chile. Ministerio de Desarrollo Social. Programa Chile Crece Contigo. [cited 2012 Oct 25 ]. Available from: http://www.crececontigo.cl/

19. Chile. Ministerio de Salud. Estrategia Nacional de Salud para el Cumplimiento de los Objetivo Sanitarios 2011-2020. [cited 2012 Oct 25]. Disponible en: http://www.minsal.gob.cl/portal/url/item/b89e911085a8 30ace040.010.1650115af.pdf

20. Minoletti A, Saxena S. Informe WHO-AIMS sobre Sistema de Salud Mental en Chile. Santiago: Organización Mundial de la Salud y Ministerio de Salud de Chile; 2006.

21. Araya R, Rojas G, Fritsch R, Gaete J, Rojas M, Simon G, et al. Treating depression in primary care in low-income women in Santiago, Chile: a randomised controlled trial. Lancet. 2003;361:995-1000.

22. Araya R, Flynn T, Rojas G, Fritsch R, Simon G. Cost-effectiveness of a primary care treatment program for depression in low-income women in: Santiago, Chile. Am J Psych. 2006;163:1379-87.

23. Alvarado R, Vega J, Sanhueza G, Muñoz MG. Evaluación del programa para la detección, diagnóstico y tratamiento integral de la depresión en atención primaria, en Chile. Rev Panam Salud Pública. 2005;18(4/5):278-86. 
24. Alvarado R, Rojas G, Minoletti A, Domínguez C, Alvarado F. Depression program in primary health care: the Chilean experience. Int J Mental Health. 2012. In press.

25. Rojas G, Fritsch R, Solis J, Jadresic E, Castillo C, Gonzalez M, et al. Treating postnatal depression among low-income mothers in primary care in Santiago, Chile: a randomized controlled trial. Lancet. 2007;370:1629-37.

26. Minoletti A, Narváez P, Sepúlveda R, Caprile A. Chile: Lecciones aprendidas en la implementación de un modelo comunitario de atención en salud mental. En: Rodríguez J (Ed.). Salud Mental en la Comunidad. Washington, DC: Organización Panamericana de la Salud serie PALTEX; 2009. p. 339-48.

27. Minoletti A, Sepúlveda R, Horvitz-Lennon M. Twenty years of mental health policies in Chile: lessons and challenges. Int J Mental Health. 2012;41(1):21-37.

Recibido en: 09/08/2012 Aprobado en: 24/10/2012 